Grand Valley State University

ScholarWorks@GVSU

\title{
A Trial Examining an Advanced Practice Nurse Intervention to Promote Medication Adherence and Symptom Management in Adult Cancer Patients Prescribed Oral Anti-Cancer Agents: Study Protocol
}

Sandra L. Spoelstra

Grand Valley State University, spoelsts@gvsu.edu

Peggy S. Burhenn

City of Hope National Medical Center

Tracy DeKoekkoek

Michigan State University

Monica Schueller

Michigan State University

Follow this and additional works at: https://scholarworks.gvsu.edu/kcon_articles

Part of the Medicine and Health Sciences Commons

\section{ScholarWorks Citation}

Spoelstra, Sandra L.; Burhenn, Peggy S.; DeKoekkoek, Tracy; and Schueller, Monica, "A Trial Examining an Advanced Practice Nurse Intervention to Promote Medication Adherence and Symptom Management in Adult Cancer Patients Prescribed Oral Anti-Cancer Agents: Study Protocol" (2016). Peer Reviewed Articles. 27.

https://scholarworks.gvsu.edu/kcon_articles/27

This Article is brought to you for free and open access by the Kirkhof College of Nursing at ScholarWorks@GVSU. It has been accepted for inclusion in Peer Reviewed Articles by an authorized administrator of ScholarWorks@GVSU.

For more information, please contact scholarworks@gvsu.edu. 


\section{JOURNAL: Journal of Advanced Nursing TITLE PAGE}

Full Title A trial examining an advanced practice nurse intervention to promote medication adherence and symptom management in adult cancer patients prescribed oral anti-cancer agents: study protocol

\section{Running head NURSE INTERVENTION ADHERENCE}

Authors Sandra L. SPOELSTRA, PhD, RN; Peggy S. BURHENN, MS, CNS, AOCNS; Tracy

DEKOEKKOEK, RN; Monica SCHUELLER, BA

\section{Author Affiliations}

Michigan State University, East Lansing, MI: College of Nursing (Dr. Spoelstra, and Ms.

DeKoekkoek and Schueller); and City of Hope National Medical Center (Ms. Burhenn).

\section{Corresponding Author}

Sandra L. Spoelstra, PhD, RN

College of Nursing, Michigan State University

1355 Bogue Street, Room C342, East Lansing, MI 48824

Email (spoelst5@msu.edu)Phone 517-353-8681

Funding Statement This research was supported by a grant entitled: An intervention to promote symptom management and oral agent adherence: ADHERE, from the Oncology Nursing Society Foundation (Grant Number Re39).

Conflict of Interest Statement The authors have no conflicts of interest to disclose.

Clinical Trials Registration ClinicalTrials.gov Identifier: NCT02337296 


\section{ABSTRACT}

Aim To report a study protocol that refines then examines feasibility, preliminary efficacy, and satisfaction of ADHERE, an intervention using motivational interviewing and brief cognitive behavioral therapy as a mechanism for goal-oriented systematic patient education to promote symptom management and adherence among cancer patients prescribed oral anti-cancer agents. Background Cancer treatment with oral anti-cancer agents shifts responsibility for managing treatment from clinicians in supervised cancer centers to patients and their caregivers. Thus, a need exists to standardize start-of-care for support patient self-management of care at home. Design A two-phase quasi-experimental sequential design with repeated measures. Methods Sixty five adult patients newly prescribed an oral anti-cancer agent will be recruited from three community cancer centers. Phase one will enroll 5 patients to refine the ADHERE intervention prior to testing, using an iterative process. After completion, Phase two will enroll 30 patients who receive usual care to examine symptoms and ahderence. Advanced practice nurses will then be trained. Then 30 patients will be enrolled in the intervention group and provided ADHERE, a 4-week intervention using semi-structured interactions (initial face-to-face session and once a week phone sessions over 3-weeks) and a Toolkit to promote selfmanagement of care. Outcome measures include: oral anti-cancer agents adherence rate, symptom presence and severity, feasibility, and satisfaction with ADHERE. This protocol was approved January 2014 and is registered at ClinicalTrials.gov (Identifier NCT02337296). Discussion This nurse-led intervention has the potential to standardize the start-of-care trainingfor the patients to self-manage when prescribed oral anti-cancer agents for treatment.

Summary Statement Why this study is needed?

- No standard of care exists for patients newly prescribed oral anti-cancer agents.

- Many cancer patients treated with oral anti-cancer agents have difficulty managing symptoms, which may lead to adverse events and difficulty adhering to the medication regimen.

- Sub-optimal adherence rates for cancer patients prescribed oral anti-cancer agents exist, which may impact cancer treatment effectiveness.

Keywords: Advanced practice registered nurse, oral anti-cancer agent, cancer, motivational interviewing, cognitive behavioral therapy, patient education, clinical trial, practice-basedresearch 


\section{INTRODUCTION}

Innovations are changing how cancer treatment is administered. Over $25 \%$ of cancer treatment is now delivered using oral anti-cancer agent (OAs) (Bestvina et al. 2014). This shift in treatment delivery places more responsibility for managing cancer treatment on patients. However, patients are known to experience symptoms from side effects of treatment and miss as much as one-third of the prescribed OA doses required for treatment of the cancer (Spoelstra $e t$ al. 2013, Bassan et al. 2014, Puts et al. 2014). Thus, a need exists to test interventions to train patients to self-manage when OAs are prescribed. This paper reports on a protocol to examine an intervention (entitled “ADHERE”). ADHERE utilizes motivational interviewing (MI) and brief cognitive behavioral therapy (CBT) along with systematic patient education (PE) provided by advanced practice registered nurses (APRNs) to train patients how to self-manage when newly prescribed OAs.

\section{Background}

Lack of adherence to OAs is a significant clinical problem that may result in treatment failure, hospitalization, and, in some instances, reduced longevity (Bassan et al. 2014, Puts et al. 2014, Given et al. 2011). In addition, $75 \%$ of cancer patients are known to have chronic conditions (Koroukian et al. 2006, Smith et al. 2008) with self-management burden. As a consequence, OA care is another responsibility on top of self-management of other conditions (Spoelstra et al. 2015).

Models to promote self-management have been widely tested and proven effective among patients with chronic diseases (Glasgow et al. 2007); and in those with cancer (Cimprich et al. 2005, Chou et al. 2007). Evidence supports empowering patients to self-manage is an important component of cancer care (McCorkle et al. 2011). 
A literature review of 64 interventions to support self-management found three types of approaches were effective (Ruppar et al. 2008). This included providing knowledge through PE; motivating patients using MI; and improving behaviors using CBT.

$\mathrm{PE}$ is defined as the process by which clinicians impart information to patients that will alter health behaviors, and improve health status or health outcomes (Rankin et al. 2005). Metaanalysis of trials using PE demonstrates the effectiveness of teaching patients information about self-management in improving health outcomes (Conn et al. 2008). However, in combination with other approaches, effectiveness is known to increase, thus PE alone may be supplemented to further support self-management.

CBT is defined as a type of psychotherapy that helps a patient change their behavior by changing the way they think and feel about things (www.cancer.gov/dictionary). Meta-analysis of the 64 interventions found that $48 \%$ of those using CBT improved self-management (Ruppar et al. 2008). Interventions that were standardized were more effective than those that were tailored (Conn et al. 2009). An OA intervention study found CBT alone did not support improved self-management (Spoelstra et al. 2013). Additionally, others have reported that a major barrier to self-management involves motivation (Possidente et al. 2005), which may be needed for more complex or challenging health conditions like OA treatment for cancer patients.

MI is defined as a focused, goal oriented, person-centered counseling method to elicit behavior change by helping explore and resolve ambivalence, by engaging intrinsic motivation (Miller \& Rollnick 2002). Studies using MI have improved self-management in patients with HIV (DiIorio et al. 2008, Ingersoll et al. 2011), osteoporosis (Cook et al. 2007, Solomon et al. 2012), epilepsy (DiIorio et al. 2009), and cardiovascular disease (Johnson et al. 2006a, Johnson et al. 2006b). Effect sizes (ES) ranged from .22 to .67. In cancer patients, using MI interventions 
reduced pain (Thomas et al. 2012), promoted colorectal and mammography screening (Wahab et al. 2008), and improved physical activity levels (Swenson et al. 2010). Some interventions have combined MI with other approaches to increase effectiveness.

Some evidence exists regarding combining approaches, like PE, CBT, or MI, to improve self-management. A Cochrane review in HIV patients found that CBT combined with MI improved self-management (Rueda et al. 2006). Similarly, a review indicated combined CBT-MI interventions elicited behavior change, improving self-management outcomes (Spoelstra et al. 2014). MI and CBT have been combined finding improvement in self-management in challenging clinical problems such as weight loss in post-surgical bariatric patients, lowering hemoglobin A1c in Type-1 diabetics, and reducing anxiety following traumatic brain injury (Stewart et al. 2010, Hsieh et al. 2012).

Finally, in regard to the mode of delivery, $\mathrm{RN}$-delivered interventions to train patients to self-manage have proven effectiveness (Conn et al. 2008, Ruppar et al. 2008). RN-delivered interventions have been found to be particularly effective at improving health outcomes for cancer patients (Sikorksii et al. 2007, Given et al. 2010).

\section{Theoretical framework guiding the study}

To examine self-management of OAs, this study uses Social Cognitive Theory to underpin the approach to improving self-efficacy (Bandura 1977). Evidence supports how improved self-efficacy supports behaviors to self-manage (DiMatteo et al. 2007). In addition, the Information, Motivation, and Behavioral skills model (IMB) to guide intervention design and testing (see Figure 1) (Fisher et al. 2002). The IMB model hypothesizes that informing, motivating, and eliciting behaviors are the key elements of self-management (Osborn et al. 2010, Zarani et al. 2010). Thus, the goal of the ADHERE intervention is to utilize APRNs to inform, 
motivate, and elicit behavioral skills through increased self-efficacy, so that patients can selfmanage care of OA treatment in the home setting.

\section{THE STUDY}

Aim

The overall aim of this study is to promote self-management among adult cancer patients newly prescribed an OA for cancer treatment. Figure 2 presents a schematic for each phase of this protocol.

\section{Objectives}

Phase 1 To refine the ADHERE intervention in five patients using an iterative approach, combining MI with CBT and PE to promote self-management of OA prior to formal testing.

Phase 2 To conduct a trial to:

1. Determine the feasibility and satisfaction of the ADHERE intervention among adult cancer participants newly prescribed OAs.

2. Determine preliminary efficacy of the ADHERE intervention on adherence rates and symptom severity for adult cancer patients newly prescribed OAs to power a larger scale clinical trial.

3. Explore conditions under which ADHERE is more effective (e.g., older, more educated, less depressive symptoms) at lowering symptom severity and promoting OA adherence.

\section{Hypotheses for Phase 2}

- Of those who are approached to participate, $80 \%$ will enroll in the study.

- Of those who are in the intervention group, $80 \%$ of the ADHERE group will complete one face-to-face and three phone call sessions. 
- Of those who are in the intervention group, $90 \%$ of the ADHERE group will be satisfied with the intervention.

- The ADHERE group will have lower symptom severity compared to the control group during weeks 2 to 8 .

- The ADHERE group will demonstrate a higher adherence rate (percentage of OAs taken over the 8-week period) compared to control group.

- The attrition rate will not exceed $25 \%$.

\section{Methodology and design}

This is a two phase practice-based clinical trial enrolling 65 patients newly prescribed OAs (see Figure 2). The project was funded by the Oncology Nursing Society Foundation in December 2013.

Phase 1 is a 4-week ( $\mathrm{n}=5$ ) study, which will be conducted to refine ADHERE. We will consecutively enroll five patients, completing one then enrolling another, refining ADHERE after each patient. This iterative design has proven to be effective in practice-based research, used since 1945 in drug testing, internal medicine, cardiology, and nutrition (Francis 2005, Newcombe 2005). Single subject design has proven effectiveness when research questions are driven by clinical work with crucial questions, such as adherence to OAs (Janosky 2005). This design supports refining the ADHERE intervention prior to testing, with limited research funds.

Phase 2 is an 8-week, two-group, prospective quasi-experimental study that will first enroll the control $(n=30)$ care group, followed by accrual of the intervention group $(n=30)$. The choice of the design was informed by the risk of contaminating the control group if parallel group design was used in our practice-based research sites. APRNs, who will be the interveners in the practice setting, routinely care for patients who would be in both the control and 
intervention groups. Once the APRN interveners are trained, risk of contamination of the control group could occur if they inadvertently use components of the ADHERE intervention when caring for a patient in the control group. The potential of contamination in the parallel group randomized design would be high, with the possibility that the control group would not be solely usual care. Thus, there will be no randomization in this protocol.

\section{Participants}

Participants will be recruited from three community cancer centers. Inclusion criteria include: those age 21 years of age or over; diagnosed with cancer; and prescribed an OA within the past 30-days. Patients must also be able to speak, read, and understand English, and be able and willing to receive phone calls. Participants who are deaf, blind, or unable to accept phone calls will be excluded. We will exclude those with cognitive impairment that limits ability to understand and answer questions, as assessed by recruiters.

\section{Sample size determination}

In this exploratory study, the sample size of 60 patients for Phase 2 was selected based on the study timeline, the number of available patients, and available research resources, not statistical power. The sample size is adequate to describe feasibility, acceptability, and satisfaction. Thus, with an assumption of $20 \%$ attrition, 48 patients will be expected in the final analysis, approximately 24 in the control and 24 in the intervention group. With the sample size of 24 per group, the effect size of .83 is detectable as statistically significant; however, the purpose of this study is not to reach statistical significance but to estimate the actual ES between groups so a future study can be powered to formally test for effectiveness.

\section{Data collection and study procedures}

Data will be collected by recruiters on screening and enrollment forms, patient report, and medical and pharmacy record review. For all study phases, APRNs will audiotape face-to- 
face and phone interactions. For phase 2, telephone surveys via trained interviewers at the university for baseline, satisfation, and exit interviews will be audio-taped. Data from the forms, face-to-face and phone interactions, and interviews will be entered and stored in the web-based Patient Reported Outcome Measurement Information System (PROMIS) (www.assessmentcenter.net).

\section{Recruitment, enrollment, and consent}

Recruiters at each site will identify eligible patients from medical records. Once identified, the recruiter will meet with eligible patients, present the study, and invite them to participate using an approved enrollment folder. For phase 1, this includes a permission to contact form, a HIPPA form, a study brochure, a self-assessment tool, and adherence calendars. For phase 2, this includes for a consent form, a HIPPA form, a study brochure, a self-assessment tool, and adherence calendars. If willing to participate, the forms will be signed, and the enrollment form will be completed by the recruiter (week 0).

\section{Baseline interview}

Trained staff at the university will conduct baseline interviews (week 1) for all participants and collect data on measures and items identified in Table 1. Interviews are expected to take 25-minutes.

\section{Phase 1}

A single-subject design will enroll 5 patients sequentially to conduct the ADHERE intervention (detailed below) over 4-weeks; and refined after each patient is complete. A limitation of single-subject-design is generalizability, which will be tested in Phase 2.

\section{Phase 2 - control group}


Usual care for all patients is provided in addition to the intervention, and consists of standard care and materials provided by the cancer center, pharmacy, primary care physician, or other health care providers. In general, this includes instructions and information on the OA regimen (amount and timing), common side effects and how to manage symptoms, general ways to remember to take their OA (calendar or pill box), medication safety (storage), and how to contact a clinician for problems that arise.

\section{Phase 2 - experimental group}

The ADHERE intervention includes a face-to-face 30-minute session with the APRN during week 1 while in the clinic/office and three subsequent, weekly (weeks 2, 3, and 4) 10minute ADHERE phone calls with the APRN to promote symptom management and adherence to their OA regimen. Semi-structured scripts, embedded with MI and CBT, will be used by the APRN for the face-to-face session (see Online Supplemental 1) and phone sessions (see Online Supplemental 2).

During the face-to-face session, the APRN will discuss and review topics about medication adherence and symptom management with the patient. The APRN will briefly explain the purpose and goal of ADHERE (see Section I). Next, the APRN will ask the patient about their past medication use patterns (see Section II). Then, APRN will discuss the importance of medication adherence and barriers and solutions to medication adherence (see Section III). The APRN will also ask the patient to recall their OA prescription (OA name, dosage, frequency, etc.). After that, APRN will provide education on OA safety tips. Next, the APRN will train the patient on how to complete the weekly assessment of their OA adherence and symptoms (see Section IV). The APRN will then select a symptom the patient has experienced, and ask if they tried any symptom management strategies at home. This will be 
followed by the APRN reviewing the patient's medication profile for any potential contraindications or drug-to-drug interactions using the App Micromedex, and contacting the prescribing physician as needed to modify the drug profile (see Section V).

In the face-to-face session, the patient will be provided a copy of a Toolkit, which has been proven effective in several trials (Given et al. 2008, Sikorskii et al. 2009), to assist in adhering to their OA and managing their symptoms (see Section VI). The Toolkit is a supplemental information source, organized into a Frequently Asked Questions format. Questions include: What is the symptom? How do people describe it? What causes it? What do people do to manage it? The patient will select a symptom to work on and the APRN and patient will use the Toolkit to select symptom management strategies for the patient to try at home. The patient will also be asked to select strategies to use to ensure they adhere to their OA. The APRN will then discuss the importance in communicating with their health care provider (see Section VII). Finally, the APRN will end the session and inform the patient when they will calling them for their follow-up ADHERE calls (see Section VII).

As follow-up to the face-to-face session, phone sessions will be conducted weekly for three weeks. During the phone sessions, the APRN will discuss with the patient if the strategies selected in the previous session were successful in managing their symptoms and OA adherence. First, the APRN will ask the patient what their thoughts were on the previous session (see Section I). Next, the APRN will review the results of the weekly assessment of their OA adherence and symptoms (see Section II). This will be followed by the APRN asking the patient if they tried any of the strategies from the previous session (see Section III). If no, the APRN will ask the patient why they did not try it, and if yes, how effective was the strategy. The APRN will then ask the patient if anything could be done differently to allow the patient to determine if they 
would like to try other strategies. Finally, the APRN will review what was discussed and inform the patient when they will call them for the follow-up call (see Section IV). In the last weekly call, the APRN will inform the patient that they can ask their oncologist or nurse for additional strategies to try.

\section{ADHERE Intervention satisfaction}

Satisfaction with the ADHERE intervention will be assessed for phase 2 patients in the experimental group by the university interviewers during week 5 , following completion of all ADHERE sessions. The experimental group will be asked about their satisfaction with the information provided by the APRN, and if they would recommend ADHERE as a way for oncologists or nurses to help patients self-manage symptoms and adhere to the OA medication. In this protocol, satisfaction will be deemed high if the scores exceed $80 \%$. The interview will be audio-recorded and is expected to last approximately 10 minutes.

\section{Weekly assessments of medication adherence}

Weekly assessments (week 2 to 7 ) will be conducted for all phase 2 patients enrolled in the study, to assess adherence to OAs and symptom severity from side effects of treatment. Patients will be provided a self-assessment form that they will complete weekly; and reported to a university interviewer by phone. The assessment was adapted using questions that were developed and tested in prior work (Spoelstra et al. 2013, Spoelstra et al. 2015). The weekly assessment are expected to take 10-minutes.

\section{Exit interviews}

Exit interviews will be conducted at the end of the study (week 8) by phone at the patient's preferred time via trained interviewers at the university. The interview will be conducted within seven days following the final weekly assessment. Exit interviews are expected 
to take 20-minutes and will be audiotaped and transcribed verbatim. See Figure 3 for measures asked during the exit interview. Data from the exit interview will be entered and stored in the PROMIS.

\section{Instruments and measures}

Factors examined in this protocol are shown in Figure 3. Patient characteristics, cancer site and stage, and OA type and regimen complexity will be collected by self-report and confirmed in the medical record by the recruiter.

Feasibility of the ADHERE intervention will be measured by the number of face-to-face and phone sessions delivered; any discontinuations during the course of the study while treatment with the OAs was ongoing; the number of patients who accepted enrollment in the study compared to the number of patients who were offered to participate; and the percent of patients who completed the study.

Depressive symptoms will be assessed using the PROMIS 8a short-form with Cronbach $\alpha$ above .85 (Pilkonis et al. 2011).Self-efficacy will be assessed using three tools: the Medication Adherence Self-efficacy Scale (MASES-R), Cronbach $\alpha 0.92$ (Ogedegbe et al. 2003); the Medication Adherence Rating Scale (MARS), Cronbach $\alpha 0.66$ (acceptable for a short 5-item scale) (Thompson et al. 2000); and the Brief Medication Questionnaire (BMQ-Specific), Cronbach $\alpha 0.77$ (Horne et al. 1999).

Symptom severity will be examined using the Symptom Experience Inventory, developed by Given and Given (Chronbach $\alpha 0.78$ ), which has been used in previous work (Given et al. 2002). For this research, 19 prevalent symptoms associated with patients undergoing treatment (fatigue, pain, skin rash/skin sores, lack of appetite, constipation, nausea or vomiting, anxiety, sleep disturbance, diarrhea, sores in mouth, shortness of breath, cough, fever of 101.0 or higher, 
joint swelling and pain, redness, peeling, or pain in the hands/feet, numbness or tingling in the hands/feet, swelling of the hands/feet, headache, and weakness) are assessed. Patients will be asked if, within the past seven days, they have experienced each symptom and, if so, to rate the severity on a 9-point scale; the scores will then be summed.

Medication adherence will be measured in three ways during the weekly phone calls and during the exit interview. First, patients will be asked to recall if they missed taking any of their OA pills in the past seven days. Second, patients will be asked to do a pill count and report the number via phone interview at all contact points. Third, the proportion of OA pills taken compared to what was prescribed will be calculated to determine the rate of adherence. Adherence values will range from $0 \%$ (completely non-adherent) to $100 \%$ (completely adherent). In addition, a cumulative determination of adherence for the period between intake and exit will be examined as an indicator of how adherence rates are related to persistence of the regimen over time.

Satisfaction with ADHERE will be measured using a tool previously developed by this research team and administered in several previous studies that had Likert-scaled items (Spoelstra et al. 2013). Items will be modified for this study by adding items describing dimensions of satisfaction with the ADHERE intervention. Satisfaction will be deemed high if the scores exceed the specified cutoff of $80 \%$.

\section{Data analysis}

Phase 1 consists of an iterative process of reviewing the intervention protocol, forms, and satisfaction of patients to refine the tools used in this study in a practice-based setting. For phase 2, descriptive statistics will be provided as appropriate for all data collected and a 95\% 
confidence interval (95\% CI) will be provided. Data analysis will be performed by using SAS 9.3. Analyses of each of the aims and hypotheses are as follows.

To address feasibility, the proportion of eligible patients who were approached compared to those who actually enrolled will be calculated and the effect size for the difference between the proportion and .80 will be evaluated. Similarly, the proportion of those who drop out of the study will be used to estimate the effect size for the proportion being below .25. To estimate the effect size, the proportion of ADHERE received by the patient over the 4-week time period will be calculated. To examine uptake, the proportion of patients who report high satisfaction, scores exceeding $80 \%$, with ADHERE will be evaluated. Since the sample size is limited in this exploratory study, the statistical tests based on the exact Binomial distribution of counts will be employed for effect size estimation. For the patients in the intervention group, logistic regression modeling will be used to explore how binary variables of use of the ADHERE intervention, continuing versus stopping the ADHERE intervention, and reporting high satisfaction relate to covariates.

Since intervention and control groups were not created using randomization, they will be compared at intake on known confounders described in measures section. If any differences between groups at intake are found, they will be included as covariates in all analyses. Further, variables determined to be associated with attrition in the analysis will be controlled for in this analysis. Distributions of the outcome variables (symptom severity and adherence) will be evaluated and normalizing transformations will be applied as needed. If normalizing transformations are not successful, then generalized linear models or generalized linear mixed effects (LME) models will be employed. All model fits will be checked using inspection of 
residuals and score tests for extra parameters. If individual outliers are detected, their influence will be evaluated by comparing models with and without outliers.

Repeated measures of symptom severity will be entered into LME or generalized LME and related to the study group, time in weeks, and covariates. The magnitude of the effect size for study group will be estimated. Percentage of pills taken will be related via a linear model or generalized linear model to study group and covariates. While the study is not formally powered for the test of hypothesis, the effect size for the intervention versus control will be estimated from this model to appropriately power future larger studies.

For the exploratory aim, models built will be extended to include, if not already in the preliminary model, measures of self-efficacy and depressive symptoms. The effects of these variables over and above study group will be evaluated to inform planning of future interventions to promote adherence and manage symptoms.

\section{Ethical considerations}

University and hospital human subject committee approvals were obtained in January 2014 (IRB\# 13-823) and this trial is registered at ClinicalTrials.gov (Identifier NCT02337296). For Phase 1 of the study, a trained recruiter will obtain permission from eligible patients for the APRN intervener to contact them regarding the study. Once permission is obtained, the APRN intervener will contact the patient for an appointment to do a home visit. The APRN will go to the home to inform, consent, and conduct the ADHERE face-to-face intervention. For Phase 2 of the study, trained recruiters at two sites will approach eligible participants, present the study, and obtain consent to participate. Participants will be provided an enrollment folder which contains a study brochure and a copy of the signed consent and HIPAA forms. Participants will be notified 
that data collected will be kept confidential, that they are able to withdraw from the study at any time, and that participation will not affect usual care.

\section{Validity and reliability}

Rigor will occur by monitoring the provision of ADHERE by digitally audio taping each session (face-to-face and phone). The investigators will evaluate fidelity and adherence to communication style and delivery of the intervention per protocol. If the communication style or delivery of the intervention is not per protocol and is not as desired, additional training will occur. Continued monitoring of taped sessions will occur until study protocol is followed. This design will allow us to carefully document the intervention and its integrity. Bellg et al. (2004) argue that fidelity is represented by how delivery, receipt, and enactment lead to outcomes. In this study, we will document ADHERE delivery and receipt (contacts, minutes, and digital recordings), and enactment (strategies used) to examine rigor. All data will be checked immediately after data collection and any errors found will be corrected by the university.

\section{DISCUSSION}

The significance of this study is that it targets a challenging clinical problem among patients with a life-threatening disease, assisting patients to lower symptom severity, improve adherence, and continue their cancer treatment (Bassan et al. 2014, Puts et al. 2014).

Responsibility for self-management and adherence is transferred to patients prescribed OAs, who have less direct contact with health care professionals (Bestvina et al. 2014). This innovative intervention involves combining three proven methods, PE, CBT, and MI, to target a difficult clinical problem, using APRNs in clinical settings where they already interact with patients on a regular basis (DiIorio et al. 2009, Given et al. 2010). This intervention will begin to standardize clinical practice and has high generalizability, as well as the potential to transform care. The 
number of cancer patients who receive treatment in pill form is increasing, and this intervention may enable them to manage their symptoms and adhere to their cancer medication, allowing them to continue their cancer treatment and achieve the optimal therapeutic response to get the most from their cancer treatment.

\section{Limitations}

Several limitations exist in this study and are acknowledged. A major limitation in this protocol is the ability to measure medication adherence, which unfortunately is the state of the science in regards to reliable measures of medication adherence. Measuring medication adherence presents several potential problems as not all dose changes are documented in pharmacy records and patient self-report may contain response bias or not be reported accurately. In addition, this is not a randomized controlled trial, powered to provide information on efficacy of the intervention. Another limitation is that potential bias may be introduced because of no randomization due to high risk of contamination. However, the bias would be ignorable if there is no association between enrollment time and study outcomes. A fourth limitation is the effect of the weekly calls to monitor symptoms and adherence, which may promote adherence in the control group, and is acknowledged. Finally, another limitation is the small sample size.

\section{CONCLUSION}

It is expected that this study will show that patients on OAs will find the intervention to be feasible and acceptable and that it will support symptom management and adherence. It is expected that participants will be satisfied with the intervention. Thus, preliminary efficacy of the ADHERE intervention will be demonstrated for cancer patients being treated with OAs. ADHERE is also easily modifiable for any type of OA and for any type of cancer. After further testing, this approach could lead to translation of findings into standardized care for cancer 
patients and improvement of clinical practice. We expect to impact patients who are on OAs, improving adherence and symptom severity.

This protocol is significant because it is a nurse-led intervention designed to be implemented directly and immediately into clinical practice at the point of care (National Institute of Nursing Research 2011). Chemotherapy classes are a common part of care for patients receiving intravenous treatment; however, assistance for patients newly prescribed OAs is underdeveloped. If the protocol aims are achieved, scientific knowledge will be advanced for medication adherence and symptom management by combining PE, CBT, and MI in one intervention (ADHERE) for patients on OAs. 
Figure 1. Synthesized social cognitive theory and Information Motivation Behavioral Skill model guiding the ADHERE intervention in this protocol

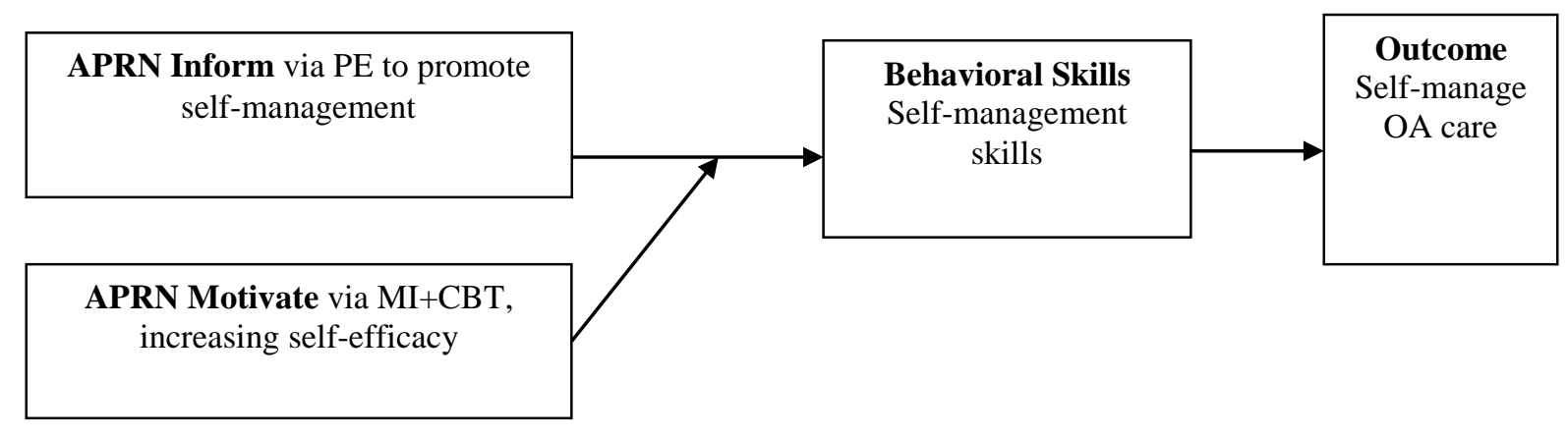


Figure 2. CONSORT schematic of steps in this protocol

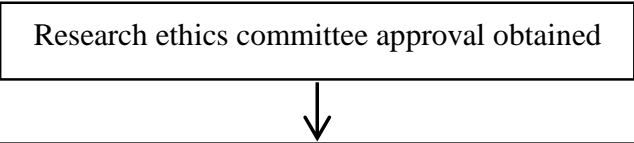

Phase 1 Week 0 Recruit, screen \& permission to contact obtained

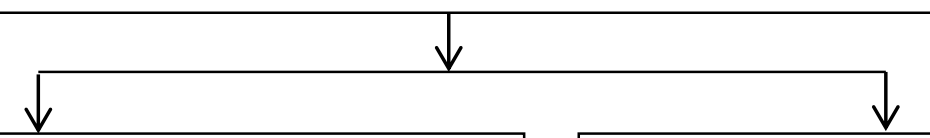

Phase 1 Enrollment (n=5) Week 1-4

Informed of study, consent; Baseline Intervie, ADHERE Face to Face and 3 phone calls; refining ADHERE intervention after each patient $x 5$

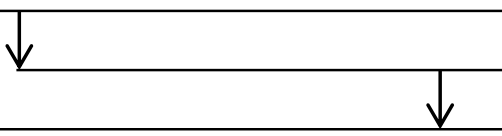

Phase 1 Week 2-7 Weekly assessment of adherence to oral agent; and symptom severity

Phase 1 Week 8 Exit interview to assess adherence to oral agent, symptom severity

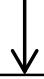

Phase 2 Control Group Week 0 (n=30) Recruit, screen \& informed of study, consent

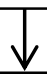

Phase 2 Control Group Week 1 Baseline interview

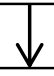

Phase 2 Control Group Week 2-7 Weekly assessment of adherence to oral agent; and symptom severity

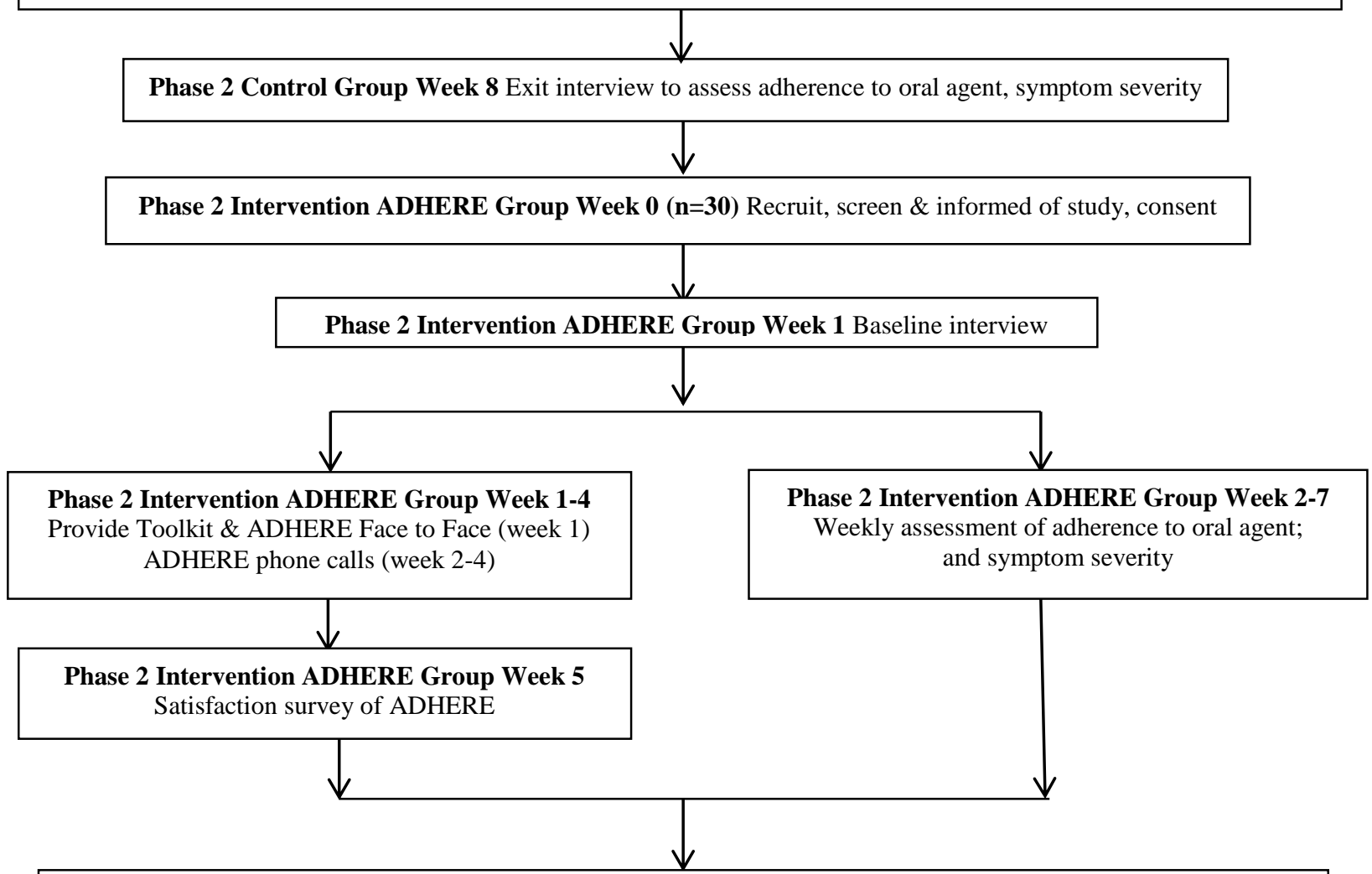

Phase 2 Intervention ADHERE Group Week 8 Exit interview to assess adherence to oral agent, symptom severity 
Table 1. Main concepts, measures, and collection schedule with protocol measurement objective

\begin{tabular}{|c|c|c|c|}
\hline Concept & Measure & Schedule & Objective \\
\hline Characteristics & Self-report and confirmed by nurse recruiter & $\begin{array}{l}\text { Phase } 1 \& 2 \\
\text { Baseline }\end{array}$ & Age, race, sex, education, \\
\hline Cancer & Diagnosed by oncologist and confirmed by nurse recruiter & $\begin{array}{l}\text { Phase } 1 \& 2 \\
\text { Baseline }\end{array}$ & Cancer site/stage \\
\hline Cancer treatment & $\mathrm{OA} /$ regimen prescribed and confirmed by nurse recruiter & $\begin{array}{l}\text { Phase } 1 \& 2 \\
\text { Baseline }\end{array}$ & Prescribed regimen dosage \\
\hline $\begin{array}{l}\text { Depressive } \\
\text { symptoms }\end{array}$ & PROMIS SF 8a Depression & $\begin{array}{l}\text { Phase } 1 \& 2 \\
\text { Baseline \& Exit }\end{array}$ & Depressive symptoms \\
\hline Self-efficacy & MASES, MARS , BMQ-Specific & $\begin{array}{l}\text { Phase } 1 \& 2 \\
\text { Baseline \& Exit }\end{array}$ & Level of self-efficacy \\
\hline $\begin{array}{l}\text { Symptom } \\
\text { severity }\end{array}$ & $\begin{array}{l}\text { Symptom Experience Inventory (yes/no) and, rate the } \\
\text { severity on a 10-point scale }\end{array}$ & $\begin{array}{l}\text { Phase } 1 \& 2 \\
\text { Baseline, weekly, exit }\end{array}$ & Level of symptom severity \\
\hline Adherence & $\begin{array}{l}\text { Patient recall, pill count of number of pills taken in the past } \\
\text { week, proportion of pills taken/compared to prescribed dose }\end{array}$ & $\begin{array}{l}\text { Phase } 1 \& 2 \\
\text { Baseline, weekly, exit }\end{array}$ & Performance of adherence \\
\hline $\begin{array}{l}\text { Feasibility \& } \\
\text { satisfaction }\end{array}$ & Recruited and enrolled and retained; satisfaction tool & $\begin{array}{l}\text { Phase } 2 \\
\text { Week } 5\end{array}$ & Feasibility \& satisfaction \\
\hline
\end{tabular}




\section{REFERENCES}

Bandura A. (1977) Self-efficacy: Toward a unifying theory of behavioral change. Psychological Review 84(2), 191-215. doi: 10.1037/0033-295x.84.2.191.

Bassan F., Peter R., Houbre B., Brennstuhl M.J., Constantini M., Speyer E. \& Tarquinio C. (2014) Adherence to oral antineoplastic agents by cancer patients: Definition and literature review. European Journal of Cancer Care 23(1), 22-35. doi: 10.1111/ecc.12124.

Bellg A.J., Borrelli B., Resnick B., Hecht J., Minicucci D.S., Ory M., Ogedegbe G., Orwig D., Ernst D., Czajkowski S. \& Treatment Fidelity Workshop of the NIH Behavior Change Consortium. (2004) Enhancing treatment fidelity in health behavior change studies: best practices and recommendations from the NIH Behavior Change Consortium. Health Psychology 23(5), 443-451. doi: 10.1037/0278-6133.23.5.443.

Bestvina C.M., Zullig L.L., Rushing C., Chino F., Samsa G.P., Altomare I., Tulsky J., Ubel P., Schrag D., Nicolla J., Abernethy A.P., Peppercorn J. \& Zafar S.Y. (2014) Patientoncologist cost communication, financial distress, and medication adherence. Journal of Oncology Practice 10(3), 162-167. doi: 10.1200/jop.2014.001406

Chou F., Dodd M., Abrams D. \& Padilla G. (2007) Symptoms, self-care, and quality of life of Chinese American patients with cancer. Oncology Nursing Forum 34(6), 1162-1167. doi: 10.1188/07.ONF.1162-1167.

Cimprich B., Janz N.K., Northouse L., Wren P.A., Given B.A. \& Given C.W. (2005) Taking charge: A self-management program for women following breast cancer treatment. Psycho-oncology 14(9), 704-717. doi: 10.1002/pon.891.

Conn V.S., Hafdahl A.R., Brown S.A. \& Brown L.M. (2008) Meta-analysis of patient education interventions to increase physical activity among chronically ill adults. Patient Education and Counseling 70(2), 157-172. doi: 10.1016/j.pec.2007.10.004.

Conn V.S., Hafdahl A.R., Cooper P.S., Ruppar T.M., Mehr D.R. \& Russell C.L. (2009) Interventions to improve medication adherence among older adults: Meta-analysis of adherence outcomes among randomized controlled trials. Gerontologist 49(4), 447-462. doi: 10.1093/geront/gnp037.

Cook P.F., Emiliozzi S. \& McCabe M. (2007) Telephone counseling to improve osteoporosis treatment adherence: An effectiveness study in community practice settings. American Journal of Medical Quality 22(6), 445-456. doi: 10.1177/1062860607307990.

DiIorio C., Escoffery C., McCarty F., Yeager K.A., Henry T.R., Koganti A., Reisinger E.L. \& Wexler B. (2009) Evaluation of WebEase: An epilepsy self-management web site. Health Education Research 24(2), 185-197. doi: 10.1093/her/cyn012.

Dilorio C., McCarty F., Resnicow K., McDonnell Holstad M., Soet J., Yeager K., Sharma S.M., Morisky D. \& Lundberg B. (2008) Using motivational interviewing to promote adherence to antiretorviral medications: A randomized control study. AIDS Care 20(3), 273-283. doi: 10.1080/09540120701593489.

DiMatteo M.R., Haskard K.B., \& Williams S.L. (2007) Health beliefs, disease severity, and patient adherence: A meta-analysis. Medical Care 45(6), 521-528. doi: 10.1097/mlr.0b013e318032937e

Fisher J.D., Fisher W.A., Bryan A.D. \& Misovich S.J. (2002) Information-motivation-behavioral skills model-based HIV risk behavior change intervention for inner-city high school youth. Health Psychology 21(2), 177-186. doi: 10.1037/0278-6133.21.2.177. 
Francis N.A. (2005) Single subject trials in primary care. Postgraduate Medical Journal 81(959), 547-548. doi: 10.1136/pgmj.2005.032581.

Given B.A., Given C.W., McCorkle R., Kozachik S., Cimprich B., Rahbar M.H. \& Wojcik C. (2002) Pain and fatigue management: Results of a nursing randomized clinical trial. Oncology Nursing Forum 29(6), 949-956. doi: 10.1188/02.ONF.949-956.

Given B.A., Spoelstra S.L. \& Grant M. (2011) The challenges of oral agents as antineoplastic treatments. Seminars in Oncology Nursing 27(2), 93-103. doi: 10.1016/j.soncn.2011.02.003.

Given C.W., Given B.A., Sikorskii A., You M., Jeon S., Champion V. \& McCorkle R. (2010) Deconstruction of nurse-delivered patient self-management interventions for symptom management: Factors related to delivery enactment and response. Annals of Behavioral Medicine 40(1), 99-113. doi: 10.1007/s12160-010-9191-7.

Given C.W., Sikorskii A., Tamkus D., Given B.A., You M., McCorkle R., Champion V. \& Decker D. (2008) Managing symptoms among patients with breast cancer during chemotherapy: Results of a two-arm behavioral trial. Journal of Clinical Oncology 26(36), 5855-5862. doi: 10.1200/jco.2008.16.8872.

Glasgow R.E., Fisher L., Skaff M., Mullan J. \& Toobert D.J. (2007) Problem solving and diabetes self-management: Investigation in a large, multiracial sample. Diabetes Care 30(1), 33-37. doi: 10.2337/dc06-1390.

Horne R., Weinman J. \& Hankins M. (1999) The beliefs about medicines questionnaire: the development and evaluation of a new method for assessing the cognitive representation of medication. Psychology and Health 14(1), 1-24. doi: 10.1080/08870449908407311.

Hsieh M-Y, Ponsford J., Wong D., Schönberger M., Taffe J. \& Mckay A. (2012) Motivational interviewing and cognitive behaviour therapy for anxiety following traumatic brain injury: A pilot randomised controlled trial. Neuropsychological Rehabilitation 22(4), 585-608. doi:10.1080/09602011.2012.678860.

Ingersoll K.S., Farrell-Carnahan L., Cohen-Filipic J., Heckman C.J., Ceperich S.D., Hettema J. \& Marzani-Nissen G. (2011) A pilot randomized clinical trial of two medication adherence and drug use interventions for HIV+ crack cocaine users. Drugs \& Alcohol Dependence 116(1-3), 177-187. doi: 10.1016/j.drugalcdep.2010.12.016.

Janosky J.E. (2005) Use of the single subject design for practice based primary care research. Postgraduate Medical Journal 81(959), 549-551. doi: 10.1136/pgmj.2004.031005.

Johnson S.S., Driskell M., Johnson J.L., Prochaska J.M., Zwick W. \& Prochaska J.O. (2006) Efficacy of a transtheoretical model-based expert system for antihypertensive adherence. Disease Management 9(5), 291-301. doi: 10.1089/dis.2006.9.291.

Johnson S.S., Driskell M.M., Johnson J.L., Dyment S.J., Prochaska J.O., Prochaska J.M. \& Bourne L. (2006) Transtheoretical model intervention for adherence to lipid-lowering drugs. Disease Management 9(2), 102-114. doi: 10.1089/dis.2006.9.102.

Koroukian S.M., Murray P. \& Madigan E. (2006) Comorbidity, disability, and geriatric syndromes in elderly cancer patients receiving home health care. Journal of Clinical Oncology 24(15), 2304-2310. doi: 10.1200/JCO.2005.03.1567.

McCorkle R., Ercolano E., Lazenby M., Schulman-Green D., Schilling L.S., Lorig K. \& Wagner E.H. (2011) Self-management: Enabling and empowering patients living with cancer as a chronic illness. CA: A Cancer Journal for Clinicians 61(1), 50-62. doi: 10.3322/caac.20093. 
Miller W.R. \& Rollnick S. (2012) Motivational interviewing: Helping people change. $3^{\text {rd }}$ Ed. New York: Guilford Press.

National Institute of Nursing Research. (2011) Bringing science to life: NINR strategic plan. [Online] Available from: http://www.ninr.nih.gov/NR/rdonlyres/8BE21801-0C52-44C29EEA-142483657FB1/0/NINR_StratPlan_F2_508.pdf.

National Cancer Institute. NCI Dictionary of Cancer Terms. [Online]. Available from: http://www.cancer.gov/dictionary?CdrID=650559

Newcombe R.G. (2005) Should the single subject design be regarded as a valid alternative to the randomised controlled trial? Postgraduate Medical Journal 81(959), 546-547. doi: 10.1136/pgmj.2004.031641.

Ogedegbe G., Mancuso C.A., Allegrante J.P. \& Charlson M.E. (2003) Development and evaluation of a medication adherence self-efficacy scale in hypertensive AfricanAmerican patients. Journal of Clinical Epidemiology 56(6), 520-529. doi: 10.1016/s0895-4356(03)00053-2.

Osborn C.Y. \& Egede L.E. (2010). Validation of an Information-Motivation-Behavioral Skills model of diabetes self-care (IMB-DSC). Patient Education and Counseling 79(1), 49-54. doi: 10.1016/j.pec.2009.07.016

Pilkonis P.A., Choi S.W., Reise S.P., Stover A.M., Riley W.T. \& Cella D. (2011) Item banks for measuring emotional distress from the Patient-Reported Outcomes Measurement Information System (PROMIS): depression, anxiety, and anger. Assessment 18(3), 263283. doi: $10.1177 / 1073191111411667$.

Possidente C.J., Bucci K.K. \& McClain W.J. (2005) Motivational interviewing: a tool to improve medication adherence? American Journal of Health-System Pharmacy 62(12), 13111314.

Puts M.T., Tu H.A., Tourangeau A., Howell D., Fitch M., Springall E. \& Alibhai S.M. (2014) Factors influencing adherence to cancer treatment in older adults with cancer: A systematic review. Annals of Oncology 25(3), 564-577. doi: 10.1093/annonc/mdt433.

Rankin S.H., Stallings K.D. \& London F. (2005) Patient education in health and illness. $5^{\text {th }}$ Ed. Philadelphia: Lippincott Williams \& Wilkins.

Rueda S., Park-Wyllie L., Bayoumi A.M., Tynan A.M., Antoniou T.A., Rourke S.B. \& Glazier R.H. (2006) Patient support and education for promoting adherence to highly active antiretroviral therapy for HIV/AIDS. The Cochrane Database of Systematic Reviews 2006(3), Article no. CD001442. doi: 10.1002/14651858.CD001442.pub2.

Ruppar T.M., Conn V.S. \& Russell C.L. (2008) Medication adherence interventions for older adults: Literature review. Research and Theory for Nursing Practice 22(2), 114-147. doi: 10.1891/0889-7182.22.2.114.

Sikorskii A., Given C.W., Given B.A, Jeon S., Decker V., Decker D., Champion V. \& McCorkle R. (2007) Symptom management for cancer patients: A trial comparing two multimodal interventions. Journal of Pain and Symptom Management 34(3), 253-264. doi: 10.1016/j.jpainsymman.2006.11.018.

Sikorskii A., Given C.W., You M., Jeon S. \& Given B.A. (2009) Response analysis for multiple symptoms revealed differences between arms of a symptom management trial. Journal of Clinical Epidemiology 62(7), 716-724. doi: 10.1016/j.jclinepi.2008.09.007.

Smith A.W., Reeve B.B., Bellizzi K.M., Harlan L.C., Klabunde C.N., Amsellem M., Bierman A.S. \& Hays R.D. (2008) Cancer, comorbidities, and health-related quality of life of older adults. Health Care Financing Review 29(4), 41-56. 
Solomon D.H., Iversen M.D., Avorn J., Gleeson T., Brookhart M.A., Patrick A.R., Rekedal L., Shrank W.H., Lii J., Losina E. \& Katz J.N. (2012) Osteoporosis telephonic intervention to improve medication regimen adherence: A large, pragmatic, randomized controlled trial. Archives of Internal Medicine 172(6), 477-483. doi: 10.1001/archinternmed.2011.1977.

Spoelstra S.L., Given B.A., Given C.W., Grant M., Sikorskii A., You M. \& Decker V. (2013) An intervention to improve adherence and management of symptoms for patients prescribed oral chemotherapy agents: An exploratory study. Cancer Nursing 36(1), 18-28. doi: 10.1097/NCC.0b013e3182551587.

Spoelstra S.L., Given C.W., Sikorskii A., Majumder A., Schueller M. \& Given B.A. (2015) Treatment with oral anticancer agents: Symptom severity and attribution, and interference with comorbidity management. Oncology Nursing Forum 42(1), 80-88. doi: 10.1188/15.ONF.42-01P.

Spoelstra S.L., Schueller M., Hilton M. \& Ridenour K. (2014) Intervention combining motivational interviewing and cognitive behaviour to promote medication adherence: A literature review. Journal of Clinical Nursing 24(9-10), 1163-1173. doi: 10.1111/jocn.12738.

Stewart K.E., Olbrisch M.E. \& Bean M.K. (2010) Back on track: Confronting post-surgical weight gain. Bariatric Nursing \& Surgical Patient Care 5(2), 179-185. doi: 10.1089/bar.2010.9920.

Swenson K.K., Nissen M.J. \& Henly S.J. (2010) Physical activity in women receiving chemotherapy for breast cancer: Adherence to a walking intervention. Oncology Nursing Forum 37(3), 321-330. doi: 10.1188/10.ONF.321-330.

Thomas M.L., Elliott J.E., Rao S.M., Fahey K.F., Paul S.M. \& Miaskowski C. (2012) A randomized, clinical trial of education or motivational-interviewing-based coaching compared to usual care to improve cancer pain management. Oncology Nursing Forum 39(1), 39-49. doi: 10.1188/12.ONF.39-49.

Thompson K., Kulkarni J. \& Sergejew A.A. (2000) Reliability and validity of a new Medication Adherence Rating Scale (MARS) for the psychoses. Schizophrenia Research 42(3), 241247. doi: 10.1016/S0920-9964(99)00130-9.

Wahab S., Menon U. \& Szalacha L. (2008) Motivational interviewing and colorectal cancer screening: A peek from the inside out. Patient Education \& Counseling 72(2), 210-217. doi: 10.1016/j.pec.2008.03.023.

Zarani F., Besharat M.A., Sadeghian S. \& Sarami G. (2010) The effectiveness of the information-motivation-behavioral skills model in promoting adherence in CABG patients. Journal of Health Psychology 15(6), 828-837. doi: 10.1177/1359105309357092 